\title{
Desenvolvimento de habilidades na formação de estudantes de Odontologia: a contribuição da Terapia Ocupacional e da Psicologia
}

Isabel Cristina Luck Coelho de Holanda*; Luara da Costa França**; Sandra Helena de Carvalho Albuquerque ${ }^{* * * ;}$; Veruska Gondim Fernandes****; Sharmênia de Araújo Soares Nuto*****

* Terapeuta Ocupacional, Docente do Curso de Odontologia, Universidade de Fortaleza

** Psicóloga, Docente do Curso de Odontologia, Universidade de Fortaleza

*** Cirurgião-dentista, Docente do Curso de Odontologia, Universidade de Fortaleza

**** Psicóloga, Docente do Curso de Odontologia, Universidade de Fortaleza

***** Cirurgiã-dentista, Docente do Curso de Odontologia da Universidade de Fortaleza, Pesquisadora da Fundação Oswaldo Cruz

Recebido em 22/04/2018. Aprovado em 13/01/2019.

\begin{abstract}
RESUMO
Relata-se a experiência de um Curso de Odontologia no desenvolvimento de habilidades psicomotoras, cognitivas e sociais dos discentes a partir da atuação interprofissional entre as áreas de Odontologia, Psicologia e Terapia Ocupacional. Descreve-se o diagnóstico e o acompanhamento do desenvolvimento dessas habilidades em atividades curriculares obrigatórias (unidades curriculares) e não obrigatórias (Projeto de Acompanhamento de Competências Técnicas de Odontologia PACTO). Verificou-se que, além do pioneirismo da inserção curricular da Psicologia e da Terapia Ocupacional no desenvolvimento de habilidades para a práxis odontológica, essa experiência visibiliza aos docentes e discentes e à comunidade acadêmica a importância dos aspectos psicomotores, cognitivos e sociais no processo de ensino-aprendizagem em Odontologia.

Descritores: Ensino. Educação Superior. Estudantes de Odontologia.
\end{abstract}




\section{INTRODUÇÃO}

A prática odontológica pressupõe várias competências, as quais envolvem múltiplos conhecimentos, manejos e comportamentos, que ajudam o profissional a desempenhar bem uma determinada função. As competências são obtidas mediante a educação acadêmica, treinamento e a experiência profissional. $\mathrm{O}$ emprego dessas competências na vida prática, ou seja, o "saber fazer", corresponde ao que denominamos de habilidade ${ }^{1}$.

Ao observar a práxis de um cirurgiãodentista, destaca-se prioritariamente a presença de habilidades psicomotoras, cognitivas e sociais. Essas habilidades são desenvolvidas especialmente durante a infância - considerando as mudanças significativas desde o nascimento até a fase da adolescência - mas sofrem transformações durante a vida do sujeito já que, os avanços das habilidades ocorrem constantemente ao longo de sua história, influenciados pelas vivências particulares de cada $u^{2}$.

A habilidade psicomotora apresenta uma relação recíproca e sistêmica entre o psiquismo e a motricidade, pois compreendemos que desde atividades simples até as mais complexas abrangem um grande número de movimentos coordenados e de alta precisão, como também, as sensações (vibrações) que induzem respostas comportamentais ${ }^{2}$. A habilidade cognitiva resulta de mecanismos do cérebro que são aprendidos em diferentes níveis, os quais estão relacionados com os processos de aprendizagem, de compreensão e de memorização de informações ${ }^{3}$. As habilidades sociais são elementos essenciais e necessários para uma relação interpessoal bem-sucedida. Suas categorias $^{4}$ são a civilidade, a comunicação verbal e não verbal, a assertividade, o comportamento empático e a flexibilidade comportamental ${ }^{5}$.

A soma dos domínios das habilidades psicomotoras, cognitivas e sociais resulta no desenvolvimento integral do sujeito, sendo influenciado por aspectos emocionais, afetivos, cognitivos, sensoriais e comportamentais, constituindo sua subjetividade. A noção de subjetividade corresponde ao modo singular de existência de cada ser, repercutindo no fazer do acadêmico ${ }^{6,7}$.

Ao executar uma ação com destreza, resolver uma situação, mesmo que pouco complexa, ou para alcançar um determinado objetivo, o indivíduo faz uso dessas habilidades ${ }^{1}$, que na prática odontológica são importantes ferramentas a serem aperfeiçoadas na formação dos que desejam a competência profissional.

O ensino baseado no desenvolvimento de habilidades articulado ao movimento de humanização na saúde tornou essencial a inclusão de novos temas na formação acadêmica dos futuros cirurgiões-dentistas, bem como o aprimoramento de estratégias inovadoras na capacitação de profissionais em formação ${ }^{8}$. Por isso, a importância do relato da experiência que está sendo desenvolvido pelo curso de Odontologia da Universidade de Fortaleza (UNIFOR).

Este curso iniciou suas atividades no ano de 1995, com uma proposta curricular tradicional. Foi somente em 2005 que se materializou o currículo integrado, em que as disciplinas das antigas especialidades clínicas foram substituídas por unidades curriculares integradas por perfil de complexidade dos pacientes $^{8}$. No entanto, a integração curricular não conseguiu superar o insatisfatório desempenho de motricidade fina dos discentes durante o treinamento profissional. Essas dificuldades, relatadas pelos docentes, especialmente, no contexto das atividades práticas, eram visíveis e muitas vezes interpretadas de maneira errônea, qualificando o aluno como inábil, imaturo ou desinteressado.

Diante desse cenário, a Coordenação do Curso de Odontologia iniciou um processo de acompanhamento dos discentes, por meio do Projeto de Acompanhamento de Competências 
Técnicas de Odontologia (PACTO), inicialmente com caráter curricular não obrigatório, em parceria com docentes das áreas de Terapia Ocupacional e Psicologia.

O PACTO teve início em agosto de 2015, em que acompanha o desenvolvimento pedagógico dos acadêmicos de Odontologia. Como ação inicial para estruturação do Projeto, a equipe que compõe o PACTO realizou as seguintes ações: (a) observação do desempenho funcional dos discentes durante o treinamento nos laboratórios e clínicas; (b) escuta junto aos docentes sobre as dificuldades na execução de procedimentos que envolvem motricidade fina; (c) análise das dificuldades, envolvendo também aspectos das habilidades psicomotoras e relacionais.

A experiência curricular não obrigatória do PACTO junto aos discentes e docentes modificou a forma de entender o desenvolvimento de habilidades, sendo gradativamente incluído na matriz curricular do curso em diversas unidades curriculares, pois a formação de um cirurgiãodentista é composta, em grande parcela, pelo desenvolvimento de técnicas com um conjunto de procedimentos a serem seguidos numa sequência de ações e comportamentos.

Ao pesquisar outras experiências, em âmbito nacional, relacionadas ao acompanhamento de habilidades psicomotoras, cognitivas e sociais do discente de Odontologia, constatamos a originalidade da inserção de docentes de Terapia Ocupacional e Psicologia.

Assim, o objetivo desse artigo é relatar a experiência do Curso de Odontologia da UNIFOR no desenvolvimento de habilidades discentes a partir da ação interprofissional entre as áreas de Odontologia, Psicologia e Terapia Ocupacional, como subsídios para o processo ensinoaprendizagem.

\section{RELATO DE EXPERIÊNCIA}

Este relato de experiência envolve a descrição da ação interprofissional nas atividades curriculares obrigatórias (unidades curriculares) e não obrigatórias (PACTO), desenvolvidas ao longo da formação acadêmica com discentes de diversos semestres.

\section{Atividades Curriculares Obrigatórias: Diagnóstico e Acompanhamento}

As unidades curriculares de Introdução à Odontologia, Pré-Clínica I e Propedêutica Clínica I realizam atividades de diagnóstico (quadro 1) e desenvolvimento de habilidades (quadro 2). No diagnóstico, os discentes executam práticas para autopercepção e os docentes observam a capacidade dos mesmos em desenvolver as diversas habilidades odontológicas. Nesse processo, o acompanhamento se dá pelo treinamento do "saber fazer" articulado à práxis odontológica.

A Introdução à Odontologia acontece no primeiro semestre, e dentre os objetivos de aprendizagens propostos, cabe ao discente "desenvolver a consciência das habilidades básicas necessárias para atuação profissional como cirurgião-dentista". Para o diagnóstico são realizadas práticas de alguns procedimentos não invasivos, em simuladores e em seus pares. Os estudantes também participam de um circuito psicomotor. Essas atividades constituem o primeiro contato com o instrumental e a vivência da consciência postural. Durante as atividades, os discentes preenchem check lists para expor o grau de dificuldades, ao mesmo tempo em que os docentes observam e também descrevem suas percepções.

Para o desenvolvimento de habilidades, a Introdução à Odontologia, possui também como um de seus objetivos de aprendizagem "capacitar para o desenvolvimento de habilidades sociais, considerando os aspectos comunicacionais e relacionais". Neste contexto, são executadas simulações, a exemplo da anamnese, quando é 
Quadro 1. Resumo das unidades curriculares que realizam atividades de diagnóstico para o desenvolvimento de habilidades psicomotoras, cognitivas e sociais

\begin{tabular}{|c|c|c|c|}
\hline Semestre & $\begin{array}{l}\text { Unidade } \\
\text { curricular }\end{array}$ & Atividades & $\begin{array}{l}\text { Áreas de } \\
\text { atuação dos } \\
\text { docentes }\end{array}$ \\
\hline $1^{\circ}$. & $\begin{array}{l}\text { Introdução à } \\
\text { Odontologia }\end{array}$ & $\begin{array}{l}\text { O perfil do cirurgião-dentista para atuar na realidade } \\
\text { brasileira } \\
\text { Por que desenvolver habilidades? } \\
\text { Circuito de desenvolvimento de habilidades psicomotoras } \\
\text { e cognitivas } \\
\text { Laboratório de comunicação e relacionamento } \\
\text { interpessoal } \\
\text { Práticas simuladas nos laboratórios e nas clínicas } \\
\text { multidisciplinar }\end{array}$ & $\begin{array}{l}\text { Odontologia } \\
\text { Psicologia } \\
\text { Terapia } \\
\text { Ocupacional }\end{array}$ \\
\hline $3^{\circ}$. & Pré-Clínica I & $\begin{array}{l}\text { Acompanhamento das atividades laboratoriais e clínicas } \\
\text { iniciais, estimulando e desenvolvendo as habilidades } \\
\text { básicas para a práxis odontológica }\end{array}$ & $\begin{array}{l}\text { Odontologia } \\
\text { Terapia } \\
\text { Ocupacional }\end{array}$ \\
\hline $4^{\circ}$. & Propedêutica I & $\begin{array}{l}\text { Acompanhamento das atividades laboratoriais e clínicas } \\
\text { iniciais, estimulando e desenvolvendo as habilidades } \\
\text { básicas para a práxis odontológica. }\end{array}$ & $\begin{array}{l}\text { Odontologia } \\
\text { Terapia } \\
\text { Ocupacional }\end{array}$ \\
\hline Todos & Livre demanda & Atividades laboratoriais e clínicas & Odontologia \\
\hline
\end{tabular}

Quadro 2. Resumo das unidades curriculares que desenvolvem habilidades psicomotoras, cognitivas e sociais com o envolvimento de docentes das áreas de Terapia Ocupacional e Psicologia

\begin{tabular}{|c|c|c|c|}
\hline Semestre & $\begin{array}{l}\text { Unidade } \\
\text { curricular }\end{array}$ & Atividades & $\begin{array}{l}\text { Áreas de } \\
\text { atuação dos } \\
\text { docentes }\end{array}$ \\
\hline $1^{\circ}$. & $\begin{array}{l}\text { Introdução à } \\
\text { Odontologia }\end{array}$ & $\begin{array}{l}\text { Simulações } \\
\text { Acompanhamento das atividades práticas de } \\
\text { desenvolvimento de habilidades }\end{array}$ & $\begin{array}{l}\text { Odontologia } \\
\text { Psicologia } \\
\text { Terapia } \\
\text { Ocupacional }\end{array}$ \\
\hline $3^{\circ}$. & Pré-Clinica I & $\begin{array}{l}\text { Acompanhamento das atividades laboratoriais e clínicas } \\
\text { iniciais }\end{array}$ & $\begin{array}{l}\text { Odontologia } \\
\text { Terapia } \\
\text { Ocupacional }\end{array}$ \\
\hline $3^{\circ}$. & $\begin{array}{l}\text { Saúde Bucal Coletiva } \\
\text { I }\end{array}$ & $\begin{array}{l}\text { Simulações } \\
\text { Laboratório de escovação }\end{array}$ & $\begin{array}{l}\text { Odontologia } \\
\text { Psicologia } \\
\text { Terapia } \\
\text { Ocupacional }\end{array}$ \\
\hline $4^{\circ}$. & Propedêutica I & $\begin{array}{l}\text { Acompanhamento das atividades laboratoriais e clínicas } \\
\text { iniciais }\end{array}$ & $\begin{array}{l}\text { Odontologia } \\
\text { Terapia } \\
\text { Ocupacional }\end{array}$ \\
\hline $4^{\circ}$. & $\begin{array}{l}\text { Clínica Odontológica } \\
\text { I }\end{array}$ & $\begin{array}{l}\text { Simulações, acompanhamento das atividades clínicas e } \\
\text { feedbacks }\end{array}$ & $\begin{array}{l}\text { Odontologia } \\
\text { Psicologia }\end{array}$ \\
\hline $5^{\circ}$. & $\begin{array}{l}\text { Psicologia do } \\
\text { Relacionamento I }\end{array}$ & $\begin{array}{l}\text { Desenvolvimento habilidades de comunicação, } \\
\text { autoconhecimento, autocontrole diante prática clínica }\end{array}$ & Psicologia \\
\hline $6^{\circ}$. & Clínica Infantil I & Simulações e feedbacks & $\begin{array}{l}\text { Odontologia } \\
\text { Psicologia }\end{array}$ \\
\hline $7^{\circ}$. & $\begin{array}{l}\text { Psicologia do } \\
\text { Relacionamento II }\end{array}$ & $\begin{array}{l}\text { Desenvolvimento de habilidades de comunicação com o } \\
\text { paciente infantil, adolescente e adulto, paciente } \\
\text { psiquiátrico. }\end{array}$ & Psicologia \\
\hline
\end{tabular}


solicitado aos discentes que realizem a entrevista inicial com paciente simulado. Para tanto, são elaborados scripts de perfil psicológico de paciente simulado que desafiam a condução da entrevista, como por exemplo um paciente transexual que teve o dente fraturado por agressão física perpetrada pelo companheiro, que está presente. Diante das simulações, o discente precisa lidar com seus pré-julgamentos, desenvolver seu controle emocional ante reações de agressão, culpa e choro. Em seguida à simulação, realiza-se feedback, no qual se debatem as possibilidades de condução e postura profissional requerida, com base em princípios ético-doutrinários da Constituição Federal de $1988^{9}$ e do Código de Ética Odontológico ${ }^{10}$.

Nas unidades curriculares de Pré-Clínica I e Propedêutica Clínica I, que compõem a matriz curricular no terceiro e quarto semestres, respectivamente, também se elabora o diagnóstico do discente em relação à capacidade de executar suas tarefas em laboratório e na clínica multidisciplinar. Essas unidades curriculares preparam o discente para o desenvolvimento das habilidades essenciais aos primeiros aprendizados da práxis odontológica.

Em Pré-Clinica I, o acadêmico se depara com o primeiro laboratório específico da Odontologia, quando são abordados temas como princípios de ergonomia aplicados à Odontologia; microbiologia da cárie dentária e da doença periodontal; prevenção e tratamento da cárie e da doença gengival. Apenas no semestre seguinte, na unidade curricular de Propedêutica Clínica I, desenvolve-se a prática em pacientes, realizando radiografias intra e extrabucais, bem como sua interpretação para a construção do diagnóstico clínico. Diante da realidade vivenciada pelos discentes e mediante observações e relatos dos mesmos, a docente terapeuta ocupacional identifica dificuldades no desempenho das atividades requeridas, estimulando o desenvolvimento de habilidades iniciais para o aprendizado da técnica.

Nas unidades curriculares de Introdução à Odontologia, Pré-Clínica I e Propedêutica Clínica I, a Terapia Ocupacional está formalmente inserida com o intuito de desenvolver e reforçar as habilidades psicomotoras (consciência corporal, visão espacial, percepção táctil, dominância manual, precisão do movimento); as habilidades cognitivas (raciocínio lógico, funções executivas planejamento, atenção e memória); e as habilidades sociais (comunicação, comportamento, tomada de decisão). Deste modo, o profissional de terapia ocupacional facilita a mudança ou a melhora de fatores que envolvem funções e estruturas do corpo, e habilidades de desempenho (motora, processual e de interação social) ${ }^{1}$.

As unidades curriculares pré-clínicas e clínicas que também desenvolvem habilidades com o acompanhamento interprofissional são: Saúde Bucal Coletiva I, Clínica Odontológica I, Psicologia do Relacionamento I, Clínica Infantil I, Psicologia do Relacionamento II (quadro 2).

Durante a unidade curricular de Saúde Bucal Coletiva I, os discentes do quarto semestre de Odontologia devem desenvolver atividades educativas em saúde bucal com escolares. Dentre os objetivos de aprendizagens propostos, cabe ao discente "comunicar-se com clareza e objetividade e com respeito cultural e empatia nas práticas de educação em saúde”. Para tanto, é proposta uma atividade com pacientes simulados, representando escolares, como por exemplo uma criança com sintomas depressivos, sofre bullying perpetrado pelos colegas, por estar submetida ao tratamento para leucemia; resistindo às atividades educativas propostas pelos acadêmicos.

Nessa mesma unidade curricular, o professor terapeuta ocupacional identificou nos acadêmicos limitações na escovação supervisionada, em função da inabilidade na 
execução de sua própria escovação. Para superação dessa dificuldade, foi desenvolvido o laboratório de escovação com os acadêmicos, antes da ida às escolas, a partir das contribuições da Terapia Ocupacional.

$\mathrm{Na}$ Clínica Odontológica I dentre os objetivos de aprendizagem estão a "realização de anamnese e exame físico para a construção do diagnóstico". Para tanto, estão presentes durante o atendimento clínico docentes da área de Psicologia, acompanhando o desenvolvimento de habilidades sociais e realizando simulações, a fim de preparar os discentes para o atendimento clínico no decorrer do semestre. As psicólogas observam a performance dos alunos durante os atendimentos e realizam feedbacks.

A Psicologia do Relacionamento I, no quinto semestre, corresponde a uma unidade curricular prática e vivencial, na qual os discentes têm espaço para discutir a relação e a comunicação com os pacientes com base em suas vivências no cotidiano da clínica. Ao longo dos encontros são propostas atividades com os objetivos de desenvolver habilidades de comunicação, autoconhecimento e autocontrole na prática clínica. Realizam-se ainda feedbacks e orientações acerca das questões atitudinais, comportamentais e éticas.

A Clínica Infantil I, inserida no sexto semestre, corresponde à primeira clínica na qual os discentes atenderão o público infantil e tem como um dos objetivos de aprendizagem "interagir com a criança e seus responsáveis, por meio de comunicação adequada, postura ética e acolhedora, com utilização de estratégias de manejo do comportamento e técnicas de abordagem que favoreçam o atendimento odontológico”. Em uma das práticas são distribuídos casos entre grupos de discentes e os mesmos elaboram simulações de atendimentos respaldados por referencial teórico da Odontopediatria. A docente psicóloga participa e avalia as apresentações na condução dos casos. Esta prática antecede o primeiro atendimento e nela são abordados temas como paciente com agitação psicomotora, deficiência auditiva, histórico de abuso sexual, pais/acompanhantes não cooperativos, criança enlutada, dentre outras situações.

Em Psicologia do Relacionamento II, do sétimo semestre, continua-se priorizando o desenvolvimento de habilidades sociais (comunicação e relacionamento). Nessa unidade curricular o enfoque é na relação com o paciente infantil, adolescente, adulto, idoso e com transtorno psiquiátrico. Os discentes também são treinados a comunicar uma má notícia oncológica, integrando horizontalmente com a unidade curricular Propedêutica Clínica III, em que são estudadas as lesões bucais. No decorrer do semestre é utilizado o recurso didático role playing $^{11}$ e outras técnicas respaldadas na psicologia positiva e comportamental, especificamente quanto ao treinamento de habilidades sociais.

As atividades curriculares obrigatórias estão referenciadas pelo arcabouço teórico da Terapia Ocupacional e Psicologia, pois a identificação de comportamentos e suas mudanças não são tarefas fáceis para o cirurgião-dentista, cuja formação raramente inclui treino observacional ou de manejo em ciências do comportamento ${ }^{12}$.

Veerkamp et al. ${ }^{13}$ estabelecem como condição necessária o treinamento especializado para identificar comportamentos, a fim de avaliar as reações dos pacientes e realizar intervenções para minimizar a ansiedade e melhorar a atuação colaborativa. Para reforçar essa tendência na formação em saúde, ressalta-se a importância do estabelecimento de um vínculo entre cirurgiãodentista e paciente para a elaboração de um plano de tratamento adequado ${ }^{14}$.

Acredita-se ser preciso desenvolver no estudante um adequado relacionamento com o 
paciente, por meio do aprimoramento das técnicas de comunicação interpessoal, sendo capaz de analisar os aspectos morais e legais para o exercício profissional digno, respeitoso, responsável, lícito e justo, pautado nos princípios básicos da cidadania. A capacidade do indivíduo em articular sentimentos, pensamentos e comportamentos, com finalidade de atingir objetivos pessoais, culturais e situacionais é uma competência conquistada por meio do treinamento de habilidades sociais ${ }^{4}$.

\section{Atividades Curriculares Não Obrigatórias: PACTO}

Além destas unidades curriculares, o PACTO recebe discentes que foram encaminhados por docentes de outros semestres, quando estes identificam no acadêmico, dificuldades para realizar atividades que requerem uma ou mais habilidades básicas (psicomotoras, cognitivas e sociais). Ao longo das unidades curriculares, o discente recebe feedbacks e quem necessita de acompanhamento mais efetivo é convidado a participar do PACTO. Em virtude do Projeto ser amplamente divulgado pelos seus participantes, docentes e secretaria do curso de Odontologia, também têm recebido discentes que desejam aprimorar suas habilidades básicas.

A partir do encaminhamento do aluno ao PACTO, aplica-se um instrumento de avaliação, realiza-se a escuta qualificada individual sobre as necessidades específicas dos interessados, e em seguida agenda-se encontros semanais individuais ou em grupo para as intervenções terapêuticas ocupacionais, escutas psicológicas e, quando necessário, encaminhamentos de diversas ordens (para atendimento psicológico, neurológico, psiquiátrico, com educador físico, entre outros).

As atividades desenvolvidas no PACTO não são obrigatórias para a integralização curricular, sendo realizadas em horários alternativos. Proporciona-se a consciência das dificuldades apresentadas e as possibilidades de cooperação e parceria entre o estudante e o Projeto.

O acompanhamento terapêutico ocupacional segue os seguintes passos: (a) identificação das dificuldades em determinada(s) habilidade(s) e sua origem; (b) desenvolvimento do potencial individual do discente no desempenho de tarefas específicas do cirurgiãodentista. Além da precisão do movimento ou da execução da tarefa, objetiva-se também a diminuição do gasto energético físico e mental, bem como do tempo utilizado no desempenho de uma ação ${ }^{1}$. O controle postural, mediante a consciência corporal, também tem sua importância durante os procedimentos, pois está intrinsecamente relacionado aos aspectos sensoriais e cognitivos ${ }^{2}$.

Todo esse processo é personalizado, desenvolvido presencialmente e associado às orientações para a realização de atividades em domicílio. Ressalta-se ainda, que são realizadas devolutivas ao aluno participante do Projeto e aos seus docentes orientadores das unidades curriculares.

O alcance do desempenho de um acadêmico durante a execução de uma determinada tarefa dependerá das capacidades básicas individuais e do treinamento de suas habilidades ${ }^{15}$. Portanto, quando se refere ao processo de aprendizagem de uma habilidade, pressupõe-se, no acadêmico, a existência de uma série de componentes envolvidos, tais como motivação, interesse, percepção e conscientização de suas necessidades.

$\mathrm{O}$ aprendizado da técnica associa-se à compreensão do manuseio dos instrumentais, do desenvolvimento da percepção tátil e da consciência de propriocepção, bem como o uso do movimento de pinça e da visão indireta, dentre outras habilidades. Frequentemente, o ensino da técnica se constrói supondo que já exista a habilidade manual desenvolvida, o que acentua 
ainda mais a dificuldade do educando ${ }^{15}$.

$\mathrm{Na}$ prática clínica, o docente consegue identificar a dificuldade em alguma habilidade básica, no entanto, não consegue fazer o diagnóstico da natureza do problema e tampouco consegue elaborar um plano de desenvolvimento da habilidade. Atrelado a isso, infelizmente, a rotina acadêmica não permite que os docentes, de forma conjunta com o aluno, possam criar estratégias de resolutividade da dificuldade ou de desenvolvimento de habilidades.

A aptidão motora é um conjunto de habilidades motoras de um indivíduo que se dá pela realização de uma ação, também motora, que tem uma finalidade específica a ser atingida, podendo subdividir-se em habilidades grossas ou finas $^{2}$. As habilidades motoras grossas utilizam grandes grupos musculares e se caracterizam em atividades como correr, pular e saltar; as habilidades finas são caracterizadas pelos movimentos dos músculos pequenos, ou seja, aqueles que requerem destreza motora e um emprego de força mínima, porém, denotam delicadeza, precisão e firmeza. Envolve os movimentos das mãos e dedos, dos pés, do rosto, da língua e dos lábios ${ }^{2}$.

Tais elaborações conceituais não fazem parte da rotina de estudos dos docentes da Odontologia, de forma que a detecção dos impedimentos do estudante passa pelo campo da percepção e não de um diagnóstico resolutivo advindo de análise teórica acerca da habilidade motora. Por isso, o campo de Terapia Ocupacional é estratégico no curso de Odontologia.

A importância das atividades desenvolvidas no PACTO é percebida pela a adesão de novos discentes a cada semestre, que desde a sua implantação tem tido um aumento gradativo de participantes. Todos os estudantes e atendimentos são cadastrados, sendo possível realizar o controle de evasão. Assim, no semestre inicial, 2015.2, participaram 21 (3\%) dos alunos matriculados, totalizando 63 atendimentos; enquanto que no semestre 2018.2 houve um aumento significativo, pois $128(17 \%)$ dos alunos matriculados participaram de 741 atendimentos.

\section{CONSIDERAÇÕES FINAIS}

O processo ensino-aprendizagem tem uma relação indissociável dos aspectos psicomotor, cognitivo, social, e esses são indispensáveis para o desenvolvimento de uma prática profissional segura. A aprendizagem também está respaldada em práticas gradativas, repetidas, que permitem ao acadêmico atingir o desempenho aspirado.

Além do pioneirismo da experiência relatada quanto à inserção curricular da Psicologia e da Terapia Ocupacional no desenvolvimento de habilidades, considera-se que essas vivências trazem benefícios tanto para os discentes e docentes quanto para a comunidade assistida.

Como indicações futuras para a consolidação da experiência almeja-se: (a) otimização do processo avaliativo contínuo das ações e resultados; (b) desenvolvimento da consciência dos discentes e docentes do processo formativo na prática odontológica; (c) visibilização dos aspectos psicomotores, cognitivos e sociais no processo de ensinoaprendizagem na Odontologia.

\section{ABSTRACT \\ Skills development in the education of Dental students: the contribution of occupational therapy and psychology}

This paper reports the experience of a School of Dentistry in the development of psychomotor, cognitive, and social skills of students, based on the interprofessional collaborative practice between the areas of dentistry, psychology and occupational therapy. It describes the diagnosis and follow-up of the development of these skills in compulsory curricular activities (curricular units) and non-compulsory ones (Project for Follow-up of Dentistry Technical Competences, known as $P A C T O)$. It was verified that, in addition 
to the pioneering curricular insertion of psychology and occupational therapy in the development of skills for dental praxis, this experience renders the psychomotor, cognitive, and social aspects of the teaching-learning process in Dentistry noticeable for teachers, students, and the academic community.

Descriptors: Teaching. Higher education. Students, Dental.

\section{REFERÊNCIAS}

1. Cavalcanti A, Silva e Dutra FCM, Elui VMC. Estrutura da prática da Terapia Ocupacional: domínio \& processo. Rev Ter Ocup Univ São Paulo. 2015; 26 (ed. especial): 1-49.

2. Rosa Neto F. Manual de avaliação motora. 2 ed. Florianópolis: DIOESC; 2014.

3. Tabaquim MLM. Desenvolvimento cognitivo. In: Rodrigues SD, Azoni CAS, Ciasca SM. (Org.). Transtornos do desenvolvimento: da identificação precoce às estratégias de intervenção. Ribeirão Preto: Book Toy; 2014. p. 75-84.

4. Del Prette ZAP, Del Prette A. Psicologia das habilidades sociais: terapia e educação. Petrópolis: Vozes; 2000.

5. Bock AMB. Psicologias: uma introdução ao estudo da psicologia. 13 ed. São Paulo: Saraiva; 2003.

6. Gonçalvez MGM. Psicologia, subjetividade e política públicas. São Paulo: Cortez; 2010.

7. Benzaquen GP, Moreci PR. Capacitação do médico para comunicar más notícias à criança. Rev Bras Educ Méd. 2008; 32(4):468-73.

8. Noro LRA, Nuto SAS, Moreira TP, Moura KS, Novais PMR. Projeto pedagógico: a construção coletiva. In: Noro LRA, org. Curso de Odontologia da UNIFOR: 10 anos ensinando e aprendendo. Fortaleza: Universidade de Fortaleza, 2005.

9. Constituição (1988). Constituição da República Federativa do Brasil. Brasília, DF: Senado Federal; 1988.

10. Código de ética (2012). Código de ética odontológico. Rio de Janeiro, RJ: Conselho Federal de Odontologia; 2012.

11. Nestel D, Tierney T. Role-play for medical students learning about communication: guidelines for maximising benefits. BMC Med Educ. 2007; 7(3):1-9.

12. Pico BF, Kopp MS. Paradigm shifts in medical and dental education: Behavioral sciences and behavioral medicine. Eur J Dent Educ. 2004;8(1): 25-31.

13. Veerkamp JSJ, Gruythuysen RJM, Van Amerongen WE, Hoogstraten J, Weerhijm KL. (1995). Dentist's ratings of child dentalpatients' anxiety. Commun Dent Oral Epidemiol. 1995; 23(6):356-9.

14. Guimaraes J. Relações paciente-profissional, o evento central das ciências da saúde. In: Marcucci G. Fundamentos de Odontologia Estomatologia. 2 ed. Santos: VitalBook, 2014. p. 39-58.

15. Miyadahira AMK. Capacidades motoras envolvidas na habilidade psicomotora da técnica de ressuscitação cardiopulmonar: subsídios para o processo ensinoaprendizagem. Rev Esc Enferm USP. 2001; 35(4):366-73.

\section{Correspondência para:}

Sharmênia de Araújo Soares Nuto

e-mail: nuto@unifor.br

Rua Olegário Memória, 4275 - casa 26, Sapiranga

60811-370 Fortaleza/CE 